Article

\title{
Fluctuations of Initial State and Event-by-Event Pseudo-Rapidity Correlations in High Energy Nuclear Collisions
}

\author{
Nassurlla Burtebayev ${ }^{1}$, Anastasiya Fedosimova ${ }^{2, *}$, Igor Lebedev ${ }^{3, * \mathbb{D}}$, Elena Dmitriyeva ${ }^{3}$, Sayora Ibraimova ${ }^{3}$ \\ and Ekaterina Bondar ${ }^{3}$ \\ 1 Institute of Nuclear Physics, Almaty 050032, Kazakhstan; nburtebayev@yandex.ru \\ 2 Faculty of Physics and Technology, Al-Farabi Kazakh National University, Almaty 050040, Kazakhstan \\ 3 Institute of Physics and Technology, Satbayev University, Almaty 050032, Kazakhstan; \\ dmitriyeva2017@mail.ru (E.D.); sayara_ibraimova@mail.ru (S.I.); grushevskaiya@bk.ru (E.B.) \\ * Correspondence: ananastasia@list.ru (A.F.); lebedev692007@yandex.ru (I.L.)
}

check for updates

Citation: Burtebayev, N.;

Fedosimova, A.; Lebedev, I.; Dmitriyeva, E.; Ibraimova, S.; Bondar,

E. Fluctuations of Initial State and Event-by-Event Pseudo-Rapidity Correlations in High Energy Nuclear Collisions. Universe 2022, 8, 67. https://doi.org/10.3390/ universe 8020067

Academic Editor: Maria Vasileiou

Received: 30 December 2021

Accepted: 19 January 2022

Published: 21 January 2022

Publisher's Note: MDPI stays neutral with regard to jurisdictional claims in published maps and institutional affiliations.

Copyright: (C) 2022 by the authors. Licensee MDPI, Basel, Switzerland. This article is an open access article distributed under the terms and conditions of the Creative Commons Attribution (CC BY) license (https:// creativecommons.org/licenses/by/ $4.0 /)$.

\begin{abstract}
The initial state, about which there is usually very little direct experimental information, leads to significant fluctuations in the distribution of secondary particles and fragments. In this paper, to estimate the initial state the fragmentation parameters of interacting nuclei are analyzed. To investigate the correlations, the Hurst method is used. A detailed study of event-by-event pseudorapidity correlations in terms of the Hurst index, multiplicity of secondary particles and target dependence has been carried out for heavy $(\mathrm{AgBr})$ and light $(\mathrm{HCNO})$ targets present in the nuclear emulsion (NIKFI BR-2) using Au-197 projectiles at 10.6 A GeV. Evidences of short-range particle correlations and cluster formation in the pseudo-rapidity space are found from our analysis. The total ensemble of events has been divided into four classes depending on the behavior of Hurst index: uncorrelated, with short-range correlations, with long-range correlations and mixed. Events of various types differ significantly in the multiplicity of secondary particles, fragmentation of the projectile nucleus, and have significant differences in the pseudo-rapidity distribution of secondary particles.
\end{abstract}

Keywords: nucleus-nucleus interactions; initial state of collision; fragmentation; Hurst index

\section{Introduction}

According to the present-day conceptions, the hadron substance transits within the interactions of the nuclei, at the high energies, into a state of the quark-gluon plasma (QGP), in which the quarks and gluons stay in a quasi-free state [1].

The study of quark-gluon plasma has mainly focused on two complementary directions. The first direction is associated with studies of interactions of heavy ions at the maximum available energies for the study of nuclear matter at very high temperatures and low baryon densities [2-4]. The second direction is focused on the search for the critical point of the phase transition of hadronic matter into the QGP state. It is assumed to be in the energy range from several $\mathrm{GeV}$ to several tens of $\mathrm{GeV}$. First, it is considered that the investigations nearby the critical point of the phase transition into the quark-gluon plasma will give a possibility to get the quality new results on the process dynamics. Second, according to the theoretical predictions, a mixed phase of the 'excited hadronic matter', which includes both the free quarks and gluons, and the protons with neutrons, must be formed within the range of the energies of 4 to $11 \mathrm{GeV}$ per nucleon [5-7]. On the other hand, a part of the community does not believe that the QGP can be obtained at $10 \mathrm{~A} \mathrm{GeV}$, and there are good MC models not requiring it $[8,9]$.

The study of multi-particle correlations and fluctuations in secondary particle distributions is often used to search for quark-gluon plasma, since the formation of secondary particles from a fireball of nuclear matter is of a collective nature [10]. The difficulty in 
identifying the formation of quark-gluon plasma is mainly because QGP is formed against an extensive background due to the usual processes of strong interaction. The initial state, about which there is usually very little direct experimental information, leads to significant fluctuations in the distribution of secondary particles and fragments [11-13].

In a central collision, the maximum number of nucleons interacts. If the collision is peripheral, then the overlap of the nuclei is incomplete, and the quark-gluon plasma is generated only by a part of the protons and neutrons from each nucleus. Therefore, the resulting fireball expands asymmetrically [14-16]. For the separation of fluctuations associated with the collision geometry, studies of event-by-event fluctuations are very popular [17-19]. The study of a single event can reveal very different physics than the analysis of averages over a large statistical ensemble. It is assumed that, analyzing the data of each individual event in details, it will be possible directly to observe the effects of the phase transition within those events in which the most favorable conditions for the generation of the quark-gluon plasma have been formed.

Additional information about the collision geometry is provided by the analysis of fragments of colliding nuclei. Nucleons of interacting nuclei can be divided into two separate categories: those that participate in an inelastic collision with at least one nucleon from the opposite nucleus (participants), and those that do not (spectators). Participants form secondary particles observed in detectors. Fragments of colliding nuclei consist of nucleon-spectators [20]. The greater the overlap of the interacting nuclei, the lower the total charge of the fragments should be. And so, fragmentation analysis should significantly improve the accuracy of estimating the parameters of the initial state of the interaction. In colliding beam experiments, information on projectile fragmentation is usually not available. Therefore, to evaluate the geometry of the collision, the accelerator experiment with the fixed target has advantages [21,22]. The fixed target mode also allows us to study rare processes and measure the parameters needed to analyze cosmic ray data [23-25].

In this work, we carried out a joint study of multi-particle correlations and eventby-event pseudo-rapidity fluctuations to search for non-statistical clusters of secondary particles. For this purpose, we analyzed both secondary particles emitted from the interaction region and fragments of the projectile-nucleus and target-nucleus. To study correlations, we used the Hurst method [26]. Analysis of the behavior of the Hurst curve makes it possible to distinguish stochastic fluctuations associated with statistical effects from correlated distributions and to estimate the "strength" and "length" of multi-particle correlations in the pseudo-rapidity distribution of secondary particles [27].

\section{Experimental Details}

The present analysis has been carried out with the data obtained from nuclear emulsion track detector. Stacks of NIKFI BR-2 emulsions have been exposed to $10.6 \mathrm{AGeV}{ }^{197} \mathrm{Au}$ beam at the BNL synchrotron [28].

In the measured interactions all charged secondary particles were classified according to the commonly accepted emulsion experiment terminology into following groups [29]: Projectile fragments, including one-charge, two-charge and multi-charge one. The multiplicity of projectile fragments is denoted by $N_{\mathrm{f}}$; Target fragments, so-called $h$-particles, consisting of fast $g$-particles (one-charge target fragments with kinetic energy greater than $26 \mathrm{MeV}$ ) and slow $b$-particles (one-charge target fragments with kinetic energy less than $26 \mathrm{MeV}$ or multi-charge target fragments). The multiplicity of target fragments is denoted by $N_{h}$; Shower particles or relativistic singly charged particle, emitted from interactions area. The multiplicity of shower particles is denoted by $n_{\mathrm{s}}$. Shower particles are also called $n_{\mathrm{s}}$ particles or $s$-particles.

One track in the emulsion corresponds to one fragment or one particle. The polar $(\Theta)$ and azimuthal $(\Psi)$ angles of all tracks were measured. The pseudo-rapidity $(\eta=-\ln [\tan \Theta / 2])$ was calculated for each shower particle. For each projectile fragment, the charge was measured. 
Studying the interaction of nuclei in the emulsion is highly informative. The high spatial resolution of the nuclear emulsion makes it possible to observe the act of collision in the $4 \pi$-geometry of the experiment. The method of nuclear emulsions makes it possible to study the parameters of fluctuations in the distribution not only of secondary particles from the interaction region but also of fragments of the target-nucleus and the projectile-nucleus in each interaction.

\section{Estimation of the Initial State of the Collision}

One of the most accurate methods for evaluating collision geometry is by analyzing fragments of colliding nuclei. Figure 1 shows a schematic representation of the interaction of nuclei with different degrees of centrality. The nucleon-participants are marked in dark color (based on geometric representations). The light color indicates spectator nucleons that form fragments of the projectile-nucleus and the target-nucleus.

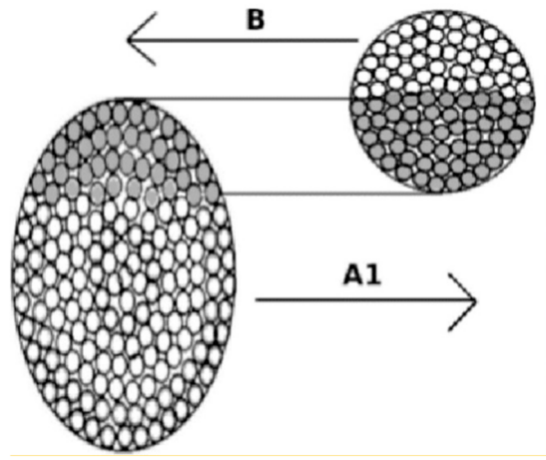

(a)

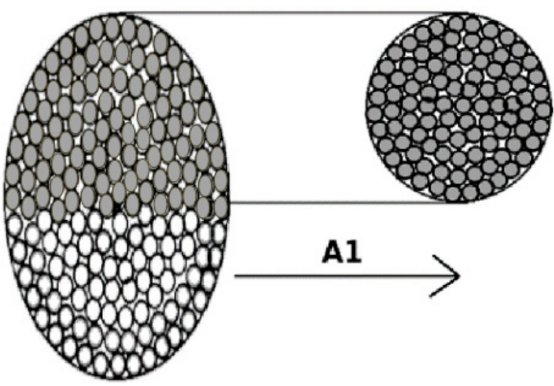

(b)

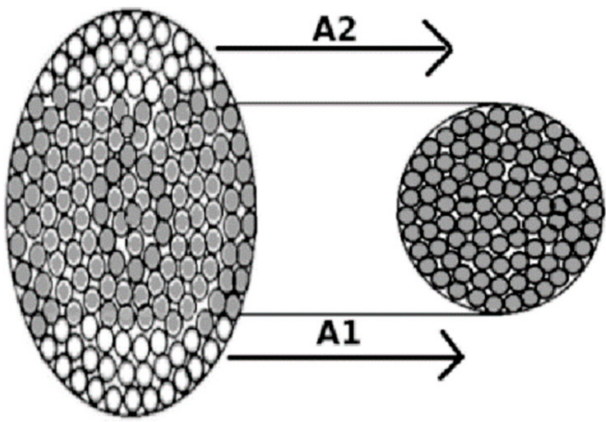

(c)

Figure 1. Schematic representation of the interaction of nuclei of various degrees of centrality: (a) peripheral interaction; (b) weakly central interaction; (c) strongly central interaction. The nucleonparticipants are marked in darker color. The light color indicates spectator nucleons that form projectile-nucleus (A1, A2) and target-nucleus (B).

Based on geometric concepts, it follows that in peripheral events (Figure 1a) one multi-charged fragment (A1) of the projectile-nucleus should be detected. Moreover, its charge is the higher, the greater the degree of collision peripherality. As well one multicharged fragment (A1) of the projectile-nucleus should be detected in weakly central interactions (Figure 1b). In strongly central interactions (Figure 1c), there should be the highest probability of occurrence of events with two multi-charged fragments (A1, A2). In this case, the smaller the target-nucleus, the larger the charges of the fragments should be detected. For large targets, events of complete destruction (when there are no multi-charged fragments) of the projectile-nucleus can be observed. Thus, depending on the peripherality of the interaction of heavy gold nuclei with different nuclei of the nuclear emulsion, a different number of multi-charged fragments is produced.

The NIKFI BR-2 emulsion includes hydrogen (39.2\%), CNO nuclei (35.3\%), and $\mathrm{AgBr}$ nuclei $(25.5 \%)$. This makes it possible to analyze nuclear interactions of different degrees of asymmetry of nuclei under the same experimental conditions.

To study the characteristics that depend on the degree of asymmetry of the nuclei, it is required to determine with which of the emulsion nuclei the projectile-nucleus interacted. The most suitable way is to analyze the number of fragments of the target-nucleus.

The separation of events with different targets was carried out according to the following criteria. First, the heaviest nucleus from $\mathrm{CNO}$ is oxygen with a charge equal to 8. Therefore, the number of fragments of the target-nucleus $\left(N_{h}\right)$ cannot be more than 8 . Second, the multiplicity of $n_{\mathrm{s}}$ in the interactions of gold with heavy nuclei $(\mathrm{Au}+\mathrm{AgBr})$ is much higher than with $\mathrm{Au}+\mathrm{CNO}$. 
Figure 2a shows the dependence of the number of fragments of the target-nucleus on the multiplicity of shower particles. The average curve for $\mathrm{Au}+\mathrm{AgBr}$ is characterized by a steady increase in the region up to $n_{\mathrm{S}} \sim 110$. In events with $n_{\mathrm{S}}>110$, with an increase in the multiplicity of $n_{\mathrm{s}}$, the number of target nucleus fragments decreases.

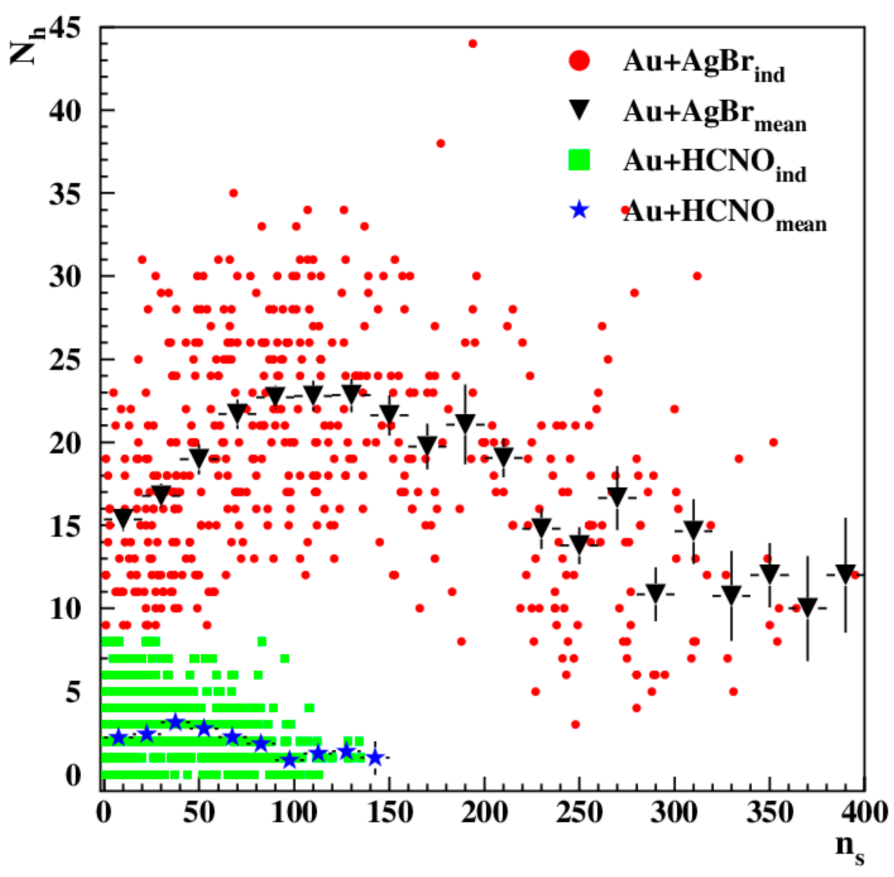

(a)
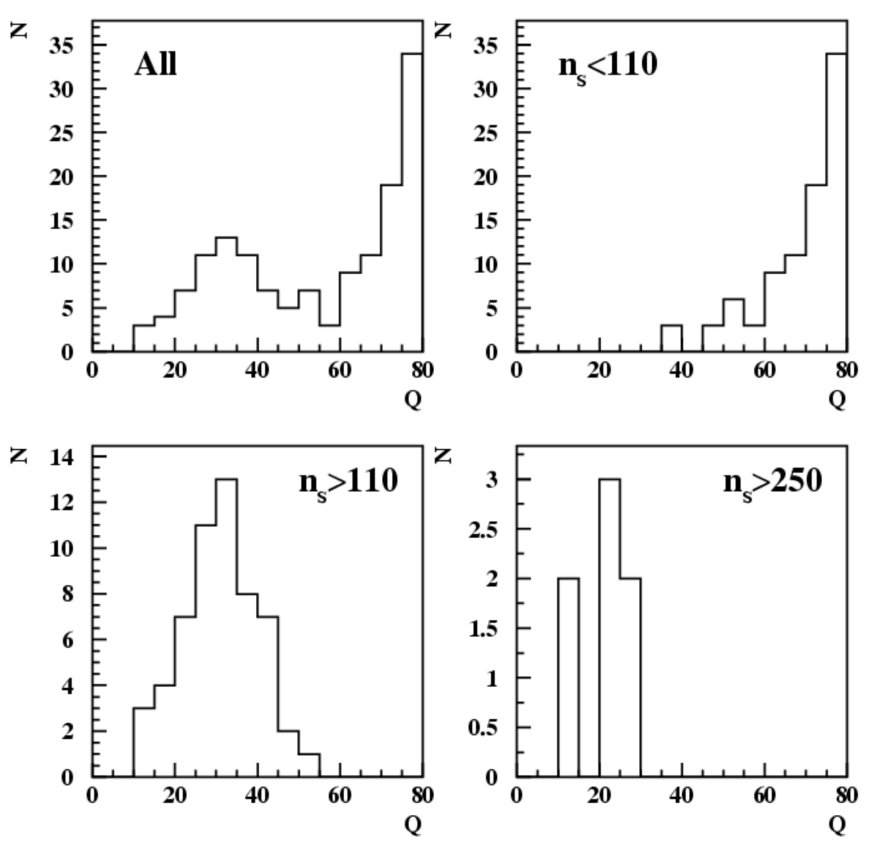

(b)

Figure 2. (a) Dependence between the number of target-nucleus fragments and the number of shower particles in the interactions of Au-197 with heavy (AgBr) and light (HCNO) emulsion nuclei at 10.6 AGeV. (b) Charge distribution of a multi-charged fragment of projectile-nucleus for the interaction of $\mathrm{Au}+\mathrm{AgBr}$ with one multi-charged fragment $N_{\mathrm{f}}=1$ depending on the $n_{\mathrm{s}}$ multiplicity.

To understand the features of this distribution, Figure $2 b$ shows the distribution of the charge of multi-charge fragments for $\mathrm{Au}+\mathrm{AgBr}$ with one multi-charged fragment for different regions of $n_{\mathrm{s}}$. The total distribution (Figure 2b-All) is characterized by two distinct peaks. The first peak is formed by events with $n_{\mathrm{s}}<110$, the second one by events with $n_{\mathrm{s}}>110$. Peripheral events of different degrees of peripherality characterize the peak in the region of large $Q$ values. The peak at $Q<55$ refers to central events and characterizes interactions with different degrees of centrality.

Based on the joint analysis of Figure $2 a, b$, the behavior of the average correlation curve is explained as follows. The level $n_{\mathrm{S}} \sim 110$ separates peripheral and central interactions. The increase in the number of $h$-particles is associated with the fragmentation of multi-charged fragments of the target-nucleus. At a level approximately corresponding to $n_{\mathrm{S}} \sim 110$, the target-nucleus is completely destroyed. At $n_{\mathrm{S}}>110$, as the centrality of the interaction increases, the multiplicity of shower particles increases. In a strongly central collision, the number of shower particles is maximal, and the number of target-nucleus fragments is minimal.

\section{Fluctuations of the Average Pseudo-Rapidity in Event}

In peripheral events (with incomplete overlapping of nuclei) the resulting fireball is taken to different directions asymmetrically. Thus, depending on the collision geometry, fluctuations of the average value of pseudo-rapidity distribution of secondary particles should be detected. To study these fluctuations in each event the average pseudo-rapidity $\langle\eta\rangle$ was calculated to search for a possible deviation of the direction of emission of sec- 
ondary particles due to the collision geometry or other reasons. Then the distribution of average pseudo-rapidity, calculated in each single event, was plotted.

Figure 3a shows the distribution of $\langle\eta\rangle$ for inelastic interactions $\mathrm{Au}+\mathrm{Em} 10.6 \mathrm{AGeV}$. As can be seen from Figure $3 a$, the $\langle\eta\rangle$ distribution is an asymmetric type. It looks like the two distributions with mean values of $\langle\eta\rangle \sim 2.35$ and $\langle\eta\rangle \sim 2.85$ overlap. For comparison in Figure $3 \mathrm{~b}$ is shown the same distribution for experimental data $\mathrm{Si}+\mathrm{Em} 14.6 \mathrm{AGeV}$ [30]. In contract to Figure $3 \mathrm{a}$, the distribution $\langle\eta\rangle$ for $\mathrm{Si}+\mathrm{Em} 14.6 \mathrm{AGeV}$ (Figure 3b) does not have a characteristic bump in the region of large $\langle\eta\rangle$.

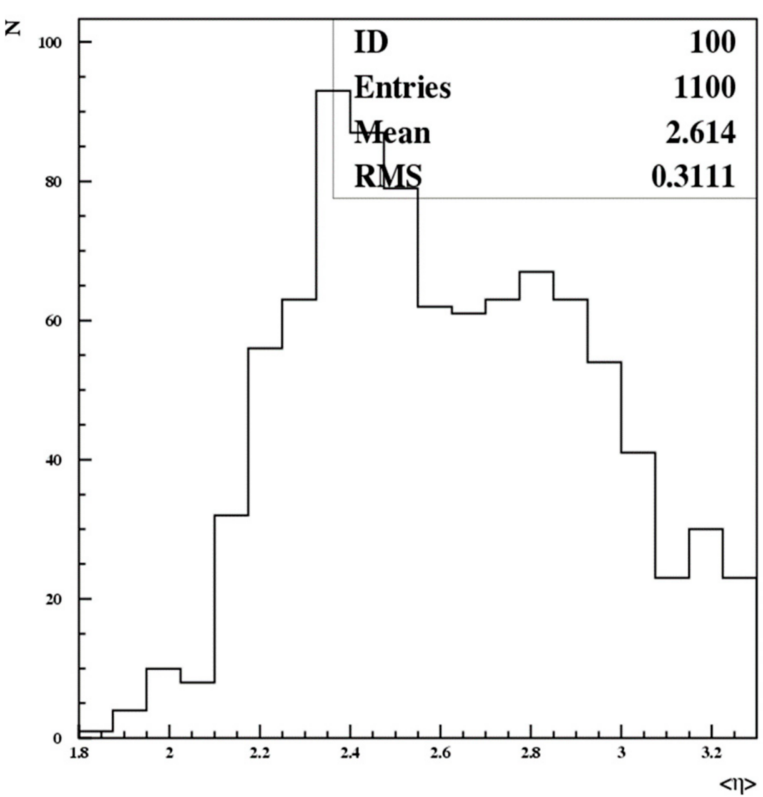

(a)

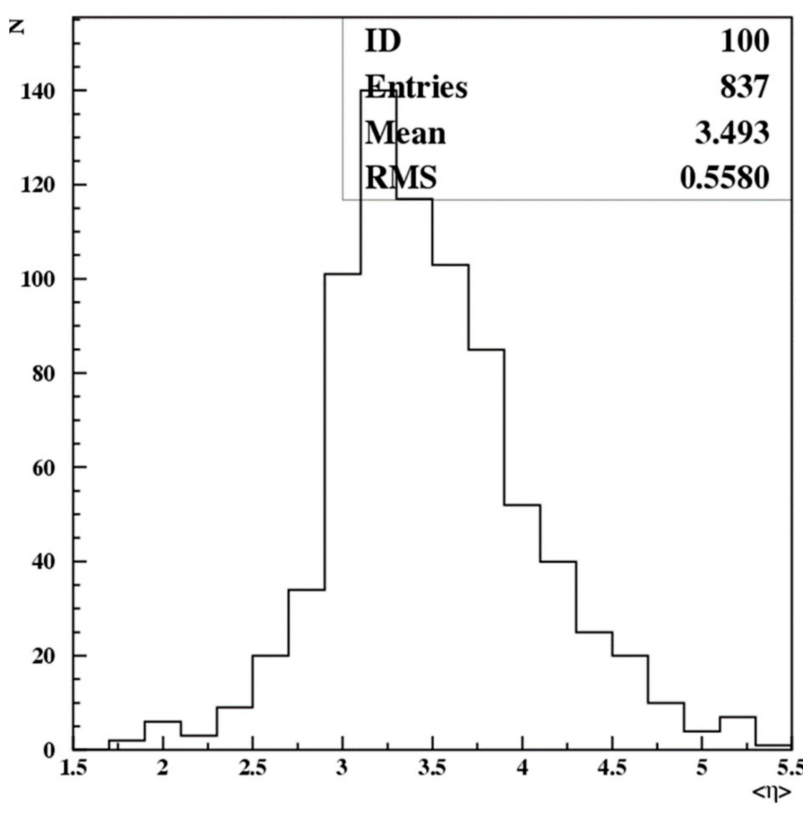

(b)

Figure 3. The distribution of the average pseudo-rapidity in event of (a) $\mathrm{Au}+\mathrm{Em} 10.6 \mathrm{AGeV}$ and (b) $\mathrm{Si}+\mathrm{Em} 14.6 \mathrm{AGeV}$.

To investigate this feature in more detail, we analyzed the pseudo-rapidity distributions of individual events, which have $\langle\eta\rangle$ in the intervals from 2.3 to 2.4 and from 2.8 to 2.9 , comparing them with each other. The distributions for four randomly selected events for each of these intervals are shown in Figure 4. As can be seen from Figure 4a, events from first interval have a Gaussian-like structure. The events from second interval (Figure $4 \mathrm{~b}$ ) have a more complex structure compared to the events shown in Figure 4a. For comparison, the same fit (of the event with the identification number $N_{\mathrm{ev}}=5517012$, normalized to the multiplicity of secondary particles in each event) by the Gaussian function is superimposed on each distribution. 

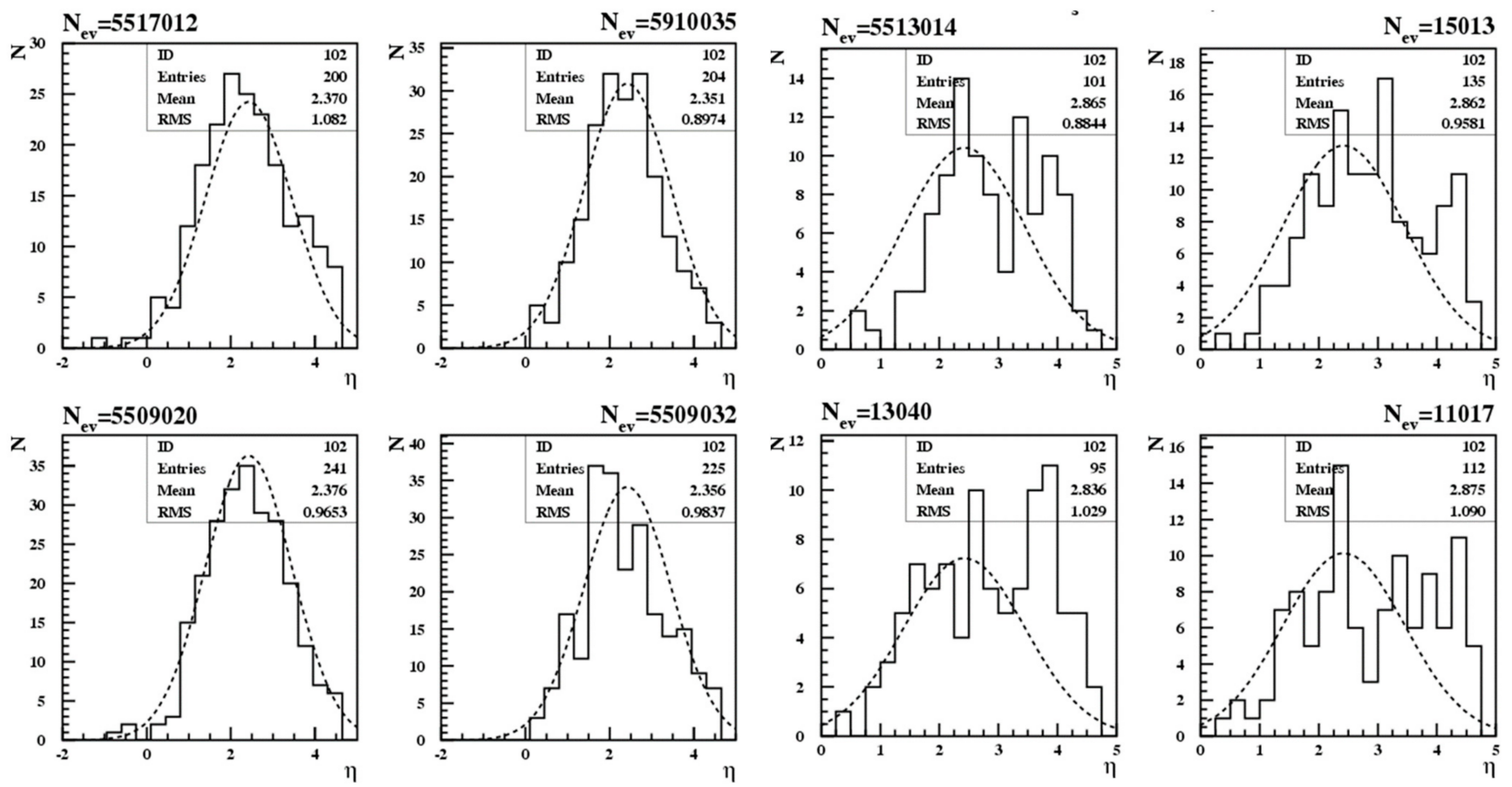

(a)

(b)

Figure 4. Pseudo-rapidity distribution of individual events $\mathrm{Au}+\mathrm{Em} 10.6 \mathrm{AGeV}$ with the mean pseudo-rapidity in the intervals: (a) from 2.3 to 2.4 and (b) from 2.8 to 2.9. Solid lines are experimental pseudo-rapidity distributions. Dashed lines are fit of the event $N_{\mathrm{ev}}=5517012$ (normalized to the multiplicity).

In the events shown in Figure $4 \mathrm{~b}$ in addition to the "standard" group of particles emitted with pseudo-rapidity $\eta \sim 2.35$, a significant part of particles have $\eta \sim 4$. Moreover, in some events, for example, $N_{\mathrm{ev}}=5,513,014$ and $N_{\mathrm{ev}}=11,017$, the distribution looks like as a two-humped distribution.

Thus, it can be stated that as a result of the interaction of gold nuclei with the nuclei of the NIKFI BR-2 emulsion in some "anomalous" events, two streams of secondary particles are formed with significantly different pseudo-rapidity. To understand whether these "anomalous" events are interactions of gold nuclei with heavy or light emulsion nuclei, we analyzed the correlations between the values of the pseudorapidity distribution of $s$-particles and the total charge of the projectile nucleus. The analysis results are shown in Figure 5. As can be seen from Figure 5 the average value of the pseudo-rapidity distribution of shower particles for the interactions of gold nuclei with $\mathrm{CNO}$ nuclei in the region $\langle\eta>\sim 2.85$. The average value of the pseudo-rapidity distribution of shower particles for interactions of gold nuclei with heavy $\mathrm{AgBr}$ nuclei is located in the region $\langle\eta\rangle \sim 2.35$. It should be noted that for the central $\mathrm{Au}+\mathrm{CNO}$ interactions of a higher degree of centrality, which are characterized by a lower value of the total charge of the residual fragments of the projectile nucleus, higher values of the average pseudo-rapidity are observed. In $\mathrm{Au}+\mathrm{AgBr}$ interactions, the opposite effect is observed: with an increase in the degree of centrality (that is, for interactions with a lower $Q$ value) of the interaction, the average pseudo-rapidity decreases. 


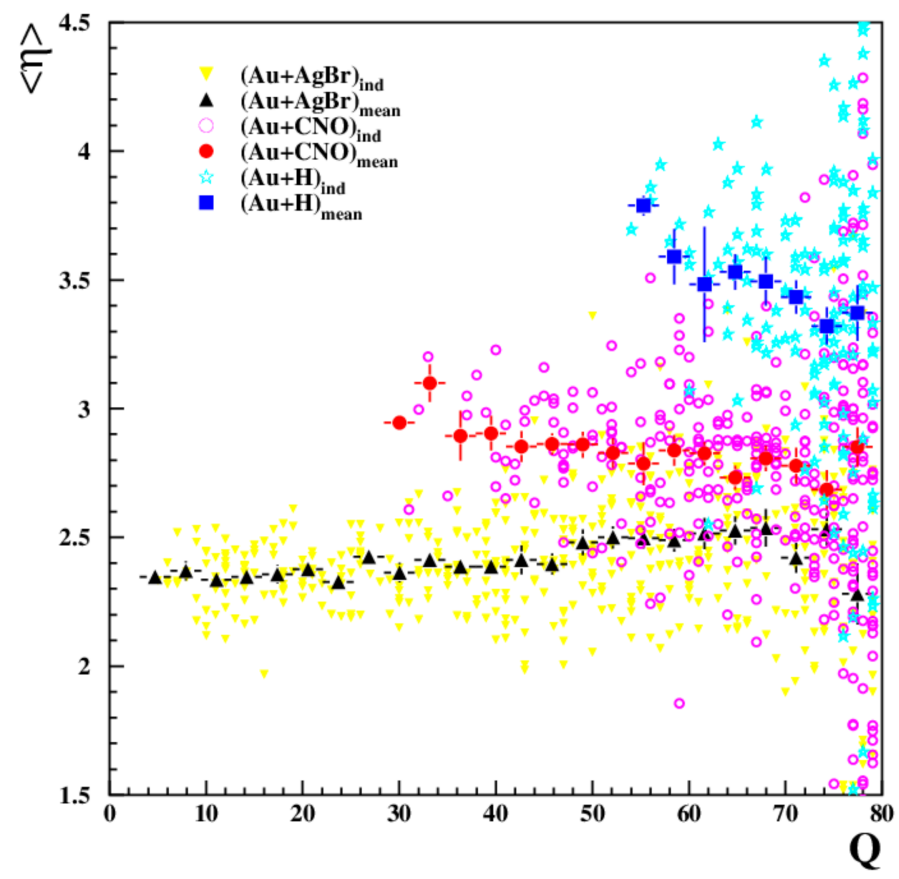

Figure 5. Average values of the pseudo-rapidity distribution of s-particles as a function of the total charge of the projectile-nucleus Au-197 with heavy and light emulsion nuclei.

The question of why the anomalous pseudo-rapidity distribution (found in $\mathrm{Au}+\mathrm{CNO}$ interactions) is not observed in $\mathrm{Si}+\mathrm{CNO}$ interactions requires careful study. This is probably due to the significantly different degree of asymmetry of the interacting nuclei. For a quantitative specification, it is convenient to use the asymmetry parameter $(L)$ that is the ratio of the number of nucleons of the projectile-nucleus to the number of nucleons of the target-nucleus. In the interactions of gold nucleus (Au-197) with silver nucleus (Ag-108), the asymmetry parameter is $L=197 / 108=1.82$. For $(\mathrm{Au}+\mathrm{O})$ interactions $L=197 / 16=12.31$. In the interactions of the silicon nucleus (Si-32) with the oxygen nucleus $L=32 / 16=2$. Thus, the asymmetry parameter of $(\mathrm{Si}+\mathrm{CNO})$ interactions is approximately equal to the asymmetry parameter of $(\mathrm{Au}+\mathrm{AgBr})$ interactions and is much smaller than $L$ for $(\mathrm{Au}+\mathrm{CNO})$ interactions.

\section{Pseudo-Rapidity Correlations}

To understand the features of the "anomalous" pseudo-rapidity distributions, the search for correlated groups of secondary particles was carried out using the Hurst method.

\subsection{Hurst Method}

The classical Hurst method was developed to analyze correlations in time sequences [26]. If some analyzed sequence $\xi_{i}$ is uncorrelated, the sum of fluctuations on a sufficiently large investigated interval of variation of the sequence $\xi_{i}(i>>1)$ will tend to zero. A correlated signal can be detected if $\Sigma\left(\xi_{i}-<\xi>\right)$ is significantly different from zero.

To quantify the degree of correlation, two basic quantities are used: the standard deviation $S(k)$ and the so-called range $R(k)$. To calculate the range, the accumulated fluctuations $X(m, k)$ of the sequence are calculated relative to the average value $\langle\xi\rangle$ :

$$
\begin{gathered}
X(m, k)=\sum_{i=1}^{m}\left[\xi_{i}-<\xi>\right], 1 \leq i \leq m \leq k \\
<\xi>=\frac{1}{k} \sum_{i=1}^{k} \xi_{i}
\end{gathered}
$$


The range is defined as the difference between the maximum and minimum values of $X(m, k)$. Next, the normalized range $H(k)$, that is the ratio of the range and standard deviation, is calculated according to the following formulas:

$$
\begin{gathered}
S(k)=\left\lceil\frac{1}{k} \sum_{i=1}^{k}\left(\xi_{i}-<\xi>\right)^{2}\right\rceil \\
R(k)=\max X(m, k)-\min X(m, k), \quad 1 \leq m \leq k \\
H(k)=R(k) / S(k)
\end{gathered}
$$

The strength and length of correlations are estimated based on the following relationship:

$$
H(k)=(a k)^{h}
$$

The parameter $a$ in Equation (6) is not used in the analysis. This is the so-called free parameter. The $h$ parameter is the Hurst index-the main parameter used to assess the strength of correlations. If the sequence is not correlated and is white noise, then the correlation index (Hurst index) will be equal to 0.5 . If the sequence $\xi_{i}$ is a fully correlated signal, then $h=1$ [31]. The strength of the signal correlation depends on the value of the Hurst index $0.5 \leq h \leq 1$. The higher the value of $h$, the more correlated the analyzed sequence is.

\subsection{Analysis Procedure}

According to modern concepts, particles from the interaction region are located in the central part of the pseudo-rapidity distribution, in the so-called pionization region. For the interactions $\mathrm{Au}+\mathrm{Em} 10.6 \mathrm{~A} \cdot \mathrm{GeV}$, the central pseudo-rapidity interval $\Delta \eta=4$ is located in the interval from $\eta_{\min }=0.3$ to $\eta_{\max }=4.3$.

For the analysis, we used fluctuations in an individual event of the relative total pseudo-rapidity distribution, normalized to the multiplicity of the s-particle. To construct the sequence $\xi_{i}$ for each event, two pseudo-rapidity distributions are used: the total pseudo-rapidity distribution of shower particles and the pseudo-rapidity distribution of an individual event. The pseudo-rapidity interval is divided into $k$ parts. We used $k=1024$ in our calculations. By counting the number of shower particles hitting each subinterval, we obtain the sequence $n_{i}^{e}$. Further, this sequence is normalized to the number of particles in this event $n^{e}$. The value $n_{i}=n_{i}^{e}$ for the full pseudo-rapidity distribution is calculated in a similar way and then normalized to $n=\sum n^{e}$. The sequences $\xi_{i}$, representing fluctuations in an individual event, is written in the following form:

$$
\xi_{i}=\left(\frac{n_{i}^{e}}{n^{e}}-\frac{n_{i}}{n}\right) / \frac{n_{i}}{n}
$$

Further, using Formulas (1)-(5), we calculated the normalized range corresponding to the length of the pseudo-rapidity interval $d \eta=4$. After that, the sequence $\xi_{i}$ was split in half into two sequences. In each of these sequences, the normalized range was calculated using Formulas (1)-(5). The value of the normalized range for these two parts was averaged and, thus, the normalized range corresponding to the length of the pseudorapidity interval $d \eta=2$ was calculated. This procedure of dividing the pseudo-rapidity interval and calculating the normalized range continued until the number of sub-intervals became less than 16, since the number of members of the analyzed sequence should be $>>1$. The obtained values of the normalized range $R / S$, depending on the length of the pseudo-rapidity interval $d \eta$ were fitted using function (6) to find the value of the Hurst index.

\subsection{Classification of Events according to the Hurst Index}

The separation of events with correlated and uncorrelated pseudo-rapidity distribution was carried out on the basis of the Hurst index $h=0.64$. The criterion $h<0.64$, as was shown 
in [32], corresponds to a pseudo-rapidity distribution in which there are no multi-particle correlations (two-particle correlations and stochastic fluctuations predominate). Based on event-by-event analysis of pseudo-rapidity correlations by the Hurst method all events were divided into 4 types: uncorrelated, with short-range correlations, with long-range correlations and mixed. Events of various types differ significantly in the fragmentation of the projectile-nucleus, multiplicity of secondary particles and pseudo-rapidity distribution. Figure 6 shows the $\mathrm{Au}+\mathrm{Em} 10.6 \mathrm{AGeV}$ events with the characteristic behavior of the Hurst curve.

(a)
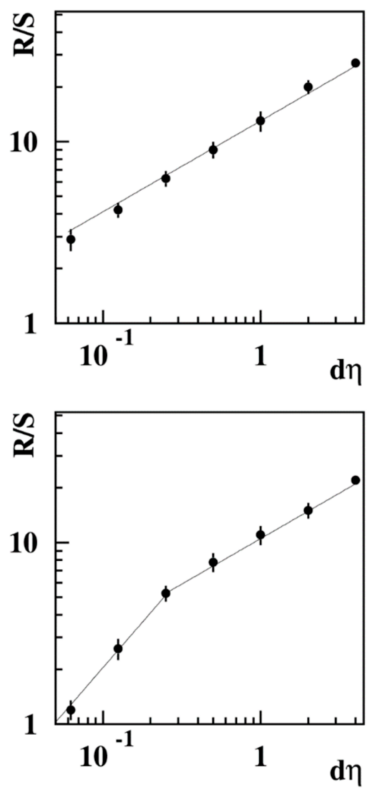

(b) (c)
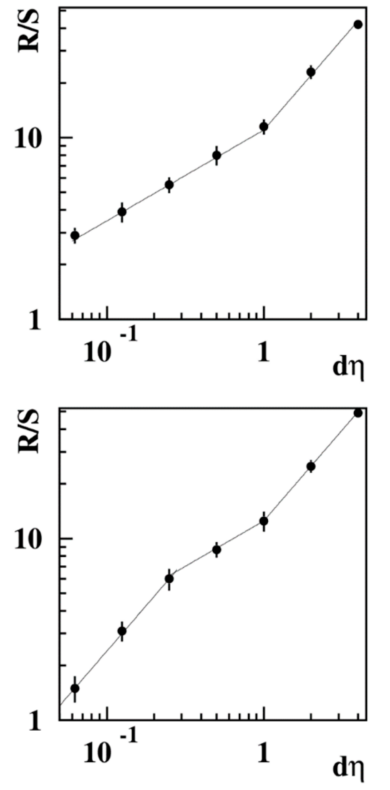

(d)

Figure 6. Behavior of the Hurst curve for four types of events Au + Em 10.6 AGeV: (a)-uncorrelated, (b) — with short-range correlations, (c)—with long-range correlations, (d)—mixed.

The first type is characterized by the linear behavior of the Hurst curve with the index $h<0.64$ (Figure 6a). This behavior of the Hurst curve corresponds to an uncorrelated pseudo-rapidity distribution. Most events of this type have one multi-charged fragment. Events of this type are most likely related to peripheral interactions.

The second type is shown in Figure 6b. The Hurst exponent in events of this type is greater than 0.64 at small pseudo-rapidity intervals $d \eta<0.25$ and less than 0.64 at pseudorapidity intervals $d \eta>0.25$. That is, short-range correlations are found in the system. In most events with short-range correlations, one multi-charged fragment of the projectile nucleus was detected. Such events are most likely to be related to central interactions with a low degree of centrality.

The third type includes the events having the Hurst index behavior shown in Figure 6c. In events of this type, $h$ is less than 0.64 at small intervals $d \eta$ and $h>0.64$ at long pseudorapidity intervals $d \eta>1$. This behavior of the Hurst curve corresponds to long-range multi-particle correlations in the pseudo-rapidity distributions of secondary particles. It evidences a cluster formation in the pseudo-rapidity space. In most events of this type, the process of complete destruction of the projectile nucleus is observed, which is characterized by the absence of multi-charged fragments of the projectile. Events with long-range correlations have the high average multiplicity $\left\langle n_{\mathrm{S}}>\sim 272\right.$. Such events mainly correspond to the central interactions of gold nuclei with heavy nuclei $(\mathrm{AgBr})$ of the emulsion.

The events shown in Figure $6 \mathrm{~d}$ have a Hurst index $h>0.64$ at small pseudo-rapidity intervals $d \eta<0.25$ and at large pseudo-rapidity intervals $d \eta>1$, but $h<0.64$ at medium pseudo-rapidity intervals. They were attributed to the fourth type and called mixed type 
events. These events have several multi-charged fragments of projectile-nucleus and mean multiplicity $<n_{\mathrm{S}}>\sim 97$. They mainly correspond to central interactions of gold nuclei with light CNO nuclei of the emulsion. Events of mixed type have "anomalous" pseudo-rapidity distribution: two streams of secondary particles are formed with significantly different pseudo-rapidity (as in Figure 4b).

\section{Conclusions}

This paper presented the joint study of multi-particle correlations and event-by-event pseudo-rapidity fluctuations in relativistic nucleus-nucleus interactions. The results were obtained on the basis of an analysis of experimental data obtained with a track detector (NIKFI BR-2) exposed to 10.6 AGeV Au-197 beam at the BNL synchrotron. Based on the analysis of the behavior of the Hurst index, the total ensemble of events has been divided into four types: uncorrelated, with short-range correlations, with long-range correlations and mixed. Events of various types differ significantly in the fragmentation of the projectile-nucleus, multiplicity of secondary particles and pseudo-rapidity distribution. Events of mixed type have "anomalous" pseudo-rapidity distribution: two streams of secondary particles are formed with significantly different pseudo-rapidity. The events mainly correspond to central interactions of heavy Au nuclei with light CNO nuclei. The revealed features of pseudo-rapidity distributions were not found in the interactions of medium Si nuclei with emulsion nuclei. This is probably due to the significantly different degree of asymmetry of the interacting nuclei.

Author Contributions: Conceptualization and methodology, I.L. and A.F.; analysis and investigation, N.B., E.D., S.I. and E.B. All authors have read and agreed to the published version of the manuscript.

Funding: This research was supported by the program \# BR10965191 (Complex Research in Nuclear and Radiation Physics, High Energy Physics and Cosmology for the Development of Competitive Technologies) of the Ministry of Education and Science of the Republic of Kazakhstan, grant number AP08855403.

Conflicts of Interest: The authors declare no conflict of interest.

\section{References}

1. Shuryak, E. Strongly coupled quark-gluon plasma in heavy ion collisions. Rev. Mod. Phys. 2017, 89, 035001. [CrossRef]

2. Abelev, B.; Adam, J.; Adamová, D.; Adare, A.; Aggarwal, M.; Rinella, G.A.; Agocs, A.; Agostinelli, A.; Salazar, S.A.; Ahammed, Z.; et al. Centrality dependence of charged particle production at large transverse momentum in $\mathrm{Pb}-\mathrm{Pb}$ collisions at $\mathrm{sNN}=2.76$ TeV. Phys. Lett. B 2013, 720, 52-62. [CrossRef]

3. Adams, J.; Aggarwal, M.M.; Ahammed, Z.; Amonett, J.; Anderson, B.D.; Arkhipkin, D.; Averichev, G.S.; Badyal, S.K.; Bai, Y.; Balewski, J.; et al. Experimental and theoretical challenges in the search for the quark-gluon plasma: The STAR Collaboration's critical assessment of the evidence from RHIC collisions. Nucl. Phys. A 2005, 757, 102-183. [CrossRef]

4. Olimov, K.K.; Liu, F.-H.; Musaev, K.A.; Olimov, A.K.; Tukhtaev, B.J.; Saidkhanov, N.S.; Yuldashev, B.S.; Olimov, K.; Gulamov, K.G. Particle species and collision energy dependencies of the midrapidity average transverse momenta of identified charged particles in $\mathrm{Au}+\mathrm{Au}$ and $\mathrm{Pb}+\mathrm{Pb}$ collisions in (Snn)1/2 = 62-5020 GeV energy range at RHIC and LHC. Int. J. Mod. Phys. E 2021, 30, 2150029. [CrossRef]

5. Ablyazimov, T.; Abuhoza, A.; Adak, R.P.; Adamczyk, M.; Agarwal, K.; Aggarwal, M.M.; Ahammed, Z.; Ahmad, F.; Ahmad, N.; Ahmad, S.; et al. Challenges in QCD matter physics-The scientific programme of the Compressed Baryonic Matter experiment at FAIR. Eur. Phys. J. A 2017, 53, 60. [CrossRef]

6. Bugaev, K.A.; Ivanytskyi, A.I.; Oliinychenko, D.R.; Sagun, V.; Mishustin, I.N.; Rischke, D.H.; Satarov, L.M.; Zinovjev, G.M. Thermodynamically anomalous regions and possible new signals of mixed-phase formation. Eur. Phys. J. A 2016, $52,175$. [CrossRef]

7. Adamczyk, L.; Adkins, J.K.; Agakishiev, G.; Aggarwal, M.M.; Ahammed, Z.; Ajitanand, N.N.; Alekseev, I.; Anderson, D.M.; Aoyama, R.; Aparin, A.; et al. Bulk properties of the medium produced in relativistic heavy-ion collisions from the beam energy scan program. Phys. Rev. C 2017, 96, 044904. [CrossRef]

8. Bopp, F.W.; Ranft, J.; Engel, R.; Roesler, S. Learning from RHIC data with DPMJET-III. Acta Phys. Polon. B 2004, $35,303-307$.

9. Capella, A.; Sukhatme, U.; Tan, C.-I.; Van, J.T.T. Dual parton model. Phys. Rep. 1994, 236, 225-329. [CrossRef]

10. Gaździcki, M.; Gorenstein, M.; Mrowczynski, S. Fluctuations and deconfinement phase transition in nucleus-nucleus collisions. Phys. Lett. B 2004, 585, 115-121. [CrossRef] 
11. Bhalerao, R.S.; Luzum, M.; Ollitrault, J.-Y. Determining initial-state fluctuations from flow measurements in heavy-ion collisions. Phys. Rev. C 2011, 84, 034910. [CrossRef]

12. Gardim, F.G.; Giacalone, G.; Luzum, M.; Ollitrault, J.-Y. Effects of initial state fluctuations on the mean transverse momentum Nucl. Phys. A 2020, 1005, 121999. [CrossRef]

13. Fedosimova, A.I.; Gaitinov, A.S.; Lebedev, I.A.; Temiraliev, A. Study on initial geometry fluctuations via correlation of finite distributions of secondary particles in nucleus-nucleus interactions. J. Phys. Conf. Ser. 2016, 668, 012067. [CrossRef]

14. Jeon, S. Initial state and flow physics-A theoretical overview. Nucl. Phys. A 2014, 932, 349-356. [CrossRef]

15. Giacalone, G.; Noronha-Hostler, J.; Ollitrault, J.-Y. Relative flow fluctuations as a probe of initial state fluctuations. Phys. Rev. C 2017, 95, 054910. [CrossRef]

16. Sarkar, S.; Mali, P.; Mukhopadhyay, A. Simulation study of elliptic flow of charged hadrons produced in Au + Au collisions at energies available at the Facility for Antiproton and Ion Research. Phys. Rev. C 2017, 95, 014908. [CrossRef]

17. Heiselberg, H. Event-by-event physics in relativistic heavy-ion collisions. Phys. Rep. 2001, 351, 161-194. [CrossRef]

18. Manna, S.K.; Mukhopadhyay, A.; Mali, P. Forward-backward multiplicity correlation and event-by-event multiplicity fluctuation in nucleus-nucleus collisions at 200A GeV. Int. J. Mod. Phys. E 2021, 30, 2150021. [CrossRef]

19. Bhattacharyya, S. Event-by-event fluctuations of maximum particle density with respect to the width of the pseudo-rapidity interval at a few A GeV/c. EPL 2020, 131, 42001. [CrossRef]

20. Alver, B.; Back, B.B.; Baker, M.D.; Ballintijn, M.; Barton, D.S.; Betts, R.R.; Bickley, A.A.; Bindel, R.; Budzanowski, A.; Busza, W.; et al. Participant and spectator scaling of spectator fragments in $\mathrm{Au}+\mathrm{Au}$ and $\mathrm{Cu}+\mathrm{Cu}$ collisions atsNN=19.6and 22.4 GeV. Phys. Rev. C 2016, 94, 024903. [CrossRef]

21. Bhattacharyya, S.; Haiduc, M.; Neagu, A.T.; Firu, E. Centrality dependence of total disintegration of target nuclei in high energy nucleus-nucleus interactions. Can. J. Phys. 2016, 94, 884-893. [CrossRef]

22. Fedosimova, A.; Gaitinov, A.; Grushevskaya, E.; Lebedev, I. Study of the peculiarities of multiparticle production via event-byevent analysis in asymmetric nucleus-nucleus interactions. EPJ Web Conf. 2017, 145, 19009. [CrossRef]

23. Kurepin, A.; Topilskaya, N. Heavy-ion collisions in a fixed target mode at the LHC beams. EPJ Web Conf. 2017, 138, 03009. [CrossRef]

24. Lebedev, I.; Fedosimova, A.; Mayorov, A.; Krassovitskiy, P.; Dmitriyeva, E.; Ibraimova, S.; Bondar, E. Direct Measurements of Cosmic Rays (TeV and beyond) Using an Ultrathin Calorimeter: Lessening Fluctuation Method. Appl. Sci. 2021, 11, 11189. [CrossRef]

25. Dmitrieva, E.; Fedosimova, A.; Lebedev, I.; Temiraliev, A.; Abishev, M.; Kozhamkulov, T.; Mayorov, A.; Spitaleri, C. Determination of the primary energy using an ultrathin calorimeter. J. Phys. G Nucl. Part. Phys. 2020, 47, 035202. [CrossRef]

26. Lloyd, E.H.; Hurst, H.E.; Black, R.P.; Simaika, Y.M. Long-Term Storage: An Experimental Study. J. R. Stat. Soc. Ser. A (Gen.) 1966, 129, 591. [CrossRef]

27. A Lebedev, I.; Shaikhatdenov, B.G. The use of the Hurst method for rapidity correlation analysis. J. Phys. G: Nucl. Part. Phys. 1997, 23, 637-641. [CrossRef]

28. Adamovich, M.; Aggarwal, M.; Alexandrov, Y.; Amirikas, R.; Andreeva, N.; Badyal, S.; Bakich, A.; Basova, E.; Bhalla, K.; Bhasin, A.; et al. Fragmentation and multifragmentation of 10.6A GeV gold nuclei. Eur. Phys. J. A 1999, 5, 429-440. [CrossRef]

29. Adamovich, M.I.; Andreeva, N.P.; Basova, E.S.; Bradnova, V.; Bubnov, V.I.; Chernyavsky, M.M.; Gaitinov, A.S.; Gulamov, K.G.; Haiduc, M.; Hasegan, D.; et al. Flow effects in high-energy nucleus collisions with $\mathrm{Ag}(\mathrm{Br})$ in emulsion. Phys. At. Nucl. 2004, 67, 273-280. [CrossRef]

30. Adamovich, M.I.; Aggarwal, M.M.; Et, A.; Andreeva, N.; Badyal, S.; Bakich, A.; Basova, E.; Bhalla, K.; Bhasin, A.; Bhatia, V.; et al. Factorial Moments of $28 \mathrm{Si}$ Induced Interactions with Ag(Br) Nuclei. Acta Physiol. Hung. 2001, 13, 213-221. [CrossRef]

31. Feder, J. Fractals, Plenum Press; Plenum Press: New York, NY, USA, 1988.

32. Kvochkina, T.N.; A Lebedev, I.; A Lebedeva, A. An analysis of high-energy interactions with large transverse momentum of secondary particles. J. Phys. G Nucl. Part. Phys. 2000, 26, 35-41. [CrossRef] 